\title{
On the integer solutions of the Pell equation $x^{2}=13 y^{2}-3^{t}$
}

\author{
V.Sangeetha $^{1} *$, M.A.Gopalan ${ }^{2}$, Manju Somanath ${ }^{3}$ \\ ${ }^{1,3}$ Assistant professor, dept. of mathematics, national college,Trichy-620001,Trichy,Tamilnadu,India \\ ${ }^{2}$ Professor,dept. of mathematics, srimathi indira Gandhi college, trichy-620002,Tamilnadu,India \\ *Corresponding author E-mail: prasansangee@gmail.com
}

Copyright $\odot 2014$ V.Sangeetha et al. This is an open access article distributed under the Creative Commons Attribution License, which permits unrestricted use, distribution, and reproduction in any medium, provided the original work is properly cited.

\begin{abstract}
The binary quadratic Diophantine equation represented by $x^{2}=13 y^{2}-3^{t}, t>0$ is considered and analyzed for its non-zero distinct integer solutions for the choices of $t$ given by (i) $t=1$ (ii) $t=3$ (iii) $t=5$ (iv) $t=2 k$ and (v) $t=2 k+5$. A few interesting relations among the solutions are presented. Further, recurrence relations on the solutions are obtained.
\end{abstract}

Keywords: Pell equation, integer solutions of Pell equation, binary quadratic Diophantine equation.

\section{Introduction}

It is well known that the Pell equation $x^{2}-D y^{2}=1$ ( $\mathrm{D}>0$ and square free) has always positive integer solutions. When $N \neq 1$, the Pell equation $x^{2}-D y^{2}=N$ may not have any positive integer solutions. For example, the equations $x^{2}=3 y^{2}-1$ and $x^{2}=7 y^{2}-4$ have no integer solutions. When $\mathrm{k}$ is a positive integer and $D \in\left(k^{2} \pm 4, k^{2} \pm 1\right)$, positive integer solutions of the equations $x^{2}-D y^{2}= \pm 4$ and $x^{2}-D y^{2}= \pm 1$ have been investigated by Jones in [9].In [3], [6], [10], [15], some specific Pell equation and their integer solutions are considered. In [1], the integer solutions of the Pell equation $x^{2}-\left(k^{2}+k\right) y^{2}=2^{t}$ has been considered. In [2], the Pell equation $x^{2}-\left(k^{2}-k\right) y^{2}=$ $2^{t}$ is analyzed for the integer solutions. In [7], the Pell equation $x^{2}-18 y^{2}=4^{k}$ is considered. In [8], the Pell equation $x^{2}-3 y^{2}=\left(k^{2}+4 k+1\right)^{t}$ is analyzed for its positive integer solutions.

This communication concerns with the Pell equation $x^{2}=13 y^{2}-3^{t}$, where $t>0$ and infinitely many positive integer solutions are obtained for the choices of $t$ given by (i) $t=1$ (ii) $t=3$ (iii) $t=5$ (iv) $t=2 k$ and (v) $t=2 k+5$.A few interesting relations among the solutions are presented. Further, recurrence relations on the solutions are derived.

\section{Notation}

$t_{4, n}=$ Square number of rank $n$.

\section{Method of analysis}

\subsection{Choice 1:t $=1$}

The Pell equation is

$$
x^{2}=13 y^{2}-3
$$

Let $\left(X_{0}, Y_{0}\right)$ be the initial solution of (1) given by

$X_{0}=7 ; Y_{0}=2$

To find the other solutions of (1), consider the Pellian equation

$x^{2}=13 y^{2}+1$

whose initial solution $\left(\tilde{x}_{n}, \tilde{y}_{n}\right)$ is given by 
$\tilde{x}_{n}=\frac{1}{2} f_{n}$

$\tilde{y}_{n}=\frac{1}{2 \sqrt{13}} g_{n}$

Where $f_{n}=(649+180 \sqrt{13})^{n+1}+(649-180 \sqrt{13})^{n+1}$

$g_{n}=(649+180 \sqrt{13})^{n+1}-(649-180 \sqrt{13})^{n+1}, n=0,1,2, \ldots$

Applying Brahmagupta lemma between $\left(X_{0}, Y_{0}\right)$ and $\left(\tilde{x}_{n}, \tilde{y}_{n}\right)$, the sequence of non-zero distinct integer solutions to $(1)$ are obtained as

$$
\begin{gathered}
X_{n+1}=\frac{1}{2}\left[7 f_{n}+2 \sqrt{13} g_{n}\right] \\
Y_{n+1}=\frac{1}{2 \sqrt{13}}\left[2 \sqrt{13} f_{n}+7 g_{n}\right]
\end{gathered}
$$

The recurrence relations satisfied by the solutions of (1) are given by

$X_{n+3}-1298 X_{n+2}+X_{n+1}=0 ; X_{1}=9223, X_{2}=11971447$

$Y_{n+3}-1298 Y_{n+2}+Y_{n+1}=0 ; Y_{1}=2558, Y_{2}=3320282$

From (2) and (3), the values of $f_{n}$ and $g_{n}$ are found to be

$f_{n}=\frac{1}{3}\left(52 Y_{n+1}-14 X_{n+1}\right) \quad ; \quad g_{n}=\frac{1}{3}\left(4 \sqrt{13} X_{n+1}-14 \sqrt{13} Y_{n+1}\right)$

\section{Properties}

1. $936 Y_{2 n+2}-252 X_{2 n+2}+108$ is a nasty number.

2. $468 Y_{3 n+3}-126 X_{3 n+3}+1404 Y_{n+1}-378 X_{n+1}$ is a cubic integer.

3. $1404 Y_{4 n+4}-378 X_{4 n+4}+324 t_{4, f_{n}}-162$ is a bi-quadratic integer.

\subsection{Choice 2: $t=3$.}

The Pell equation is

$x^{2}=13 y^{2}-27$

Let $\left(X_{0}, Y_{0}\right)$ be the initial solution of (5) given by

$X_{0}=5 ; \quad Y_{0}=2$

Applying Brahmagupta lemma between $\left(X_{0}, Y_{0}\right)$ and $\left(\tilde{x}_{n}, \tilde{y}_{n}\right)$, the sequence of non-zero distinct integer solutions to (5) are obtained as

$$
\begin{gathered}
X_{n+1}=\frac{1}{2}\left[5 f_{n}+2 \sqrt{13} g_{n}\right] \\
Y_{n+1}=\frac{1}{2 \sqrt{13}}\left[2 \sqrt{13} f_{n}+5 g_{n}\right]
\end{gathered}
$$

The recurrence relations satisfied by the solutions of (5) are given by

$X_{n+3}-1298 X_{n+2}+X_{n+1}=0 ; X_{1}=7925, X_{2}=10286645$

$Y_{n+3}-1298 Y_{n+2}+Y_{n+1}=0 ; Y_{1}=2198, Y_{2}=2853002$

From (6) and (7), the values of $f_{n}$ and $g_{n}$ are found to be

$$
f_{n}=\frac{1}{27}\left(52 Y_{n+1}-10 X_{n+1}\right) \quad ; \quad g_{n}=\frac{1}{27}\left(4 \sqrt{13} X_{n+1}-10 \sqrt{13} Y_{n+1}\right)
$$

\section{Properties}

1. $6\left(468 Y_{2 n+2}-90 X_{2 n+2}+1458\right)$ is a nasty number.

2. $468 Y_{3 n+3}-90 X_{3 n+3}+468 Y_{n+1}-90 X_{n+1}$ is a cubic integer.

3. $52 Y_{4 n+4}-10 X_{4 n+4}+324 t_{4, f_{n}}-162$ is a bi-quadratic integer.

\subsection{Choice 3: $t=5$}

The Pell equation is

$$
x^{2}=13 y^{2}-243
$$

Let $\left(X_{0}, Y_{0}\right)$ be the initial solution of (9) given by

$X_{0}=15 ; Y_{0}=6$

Applying Brahmagupta lemma between $\left(X_{0}, Y_{0}\right)$ and $\left(\tilde{x}_{n}, \tilde{y}_{n}\right)$, the sequence of non-zero distinct integer solutions to (9) are obtained as

$$
\begin{gathered}
X_{n+1}=\frac{1}{2}\left[15 f_{n}+6 \sqrt{13} g_{n}\right] \\
Y_{n+1}=\frac{1}{2 \sqrt{13}}\left[6 \sqrt{13} f_{n}+15 g_{n}\right]
\end{gathered}
$$

The recurrence relations satisfied by the solutions of (9) are given by

$X_{n+3}-1298 X_{n+2}+X_{n+1}=0 ; X_{1}=23775, X_{2}=30859935$

$Y_{n+3}-1298 Y_{n+2}+Y_{n+1}=0 ; \quad Y_{1}=6594, Y_{2}=8559006$

From (10) and (11), the values of $f_{n}$ and $g_{n}$ are found to be 
$f_{n}=\frac{1}{243}\left(156 Y_{n+1}-30 X_{n+1}\right) ; g_{n}=\frac{1}{243}\left(12 \sqrt{13} X_{n+1}-30 \sqrt{13} Y_{n+1}\right)$

\section{Properties}

1. $\left.\quad 104 Y_{2 n+2}-20 X_{2 n+2}+108\right)$ is a nasty number.

2. $52 Y_{3 n+3}-10 X_{3 n+3}+156 Y_{n+1}-30 X_{n+1}$ is a cubic integer.

3. $156 Y_{4 n+4}-30 X_{4 n+4}+324 t_{4, f_{n}}-162$ is a bi-quadratic integer

\subsection{Choice 4: $t=2 k, k>0$.}

The Pell equation is

$x^{2}=13 y^{2}-3^{2 k}, k>0$

Let $\left(X_{1}, Y_{1}\right)$ be the initial solution of (13) given by

$X_{1}=3^{k} .649 ; \quad Y_{1}=3^{k} .180$

Applying Brahmagupta lemma between $\left(X_{1}, Y_{1}\right)$ and $\left(\tilde{x}_{n}, \tilde{y}_{n}\right)$, the sequence of non-zero distinct integer solutions to (13) are obtained as

$$
\begin{gathered}
X_{n+1}=3^{k} \cdot \frac{1}{2} f_{n} \\
Y_{n+1}=3^{k} \cdot \frac{1}{2 \sqrt{13}} g_{n}, n=1,2,3, \ldots
\end{gathered}
$$

The recurrence relations satisfied by the solutions of (13) are given by

$X_{n+3}-1298 X_{n+2}+X_{n+1}=0 ; X_{2}=3^{k} .842401, X_{3}=3^{k} .1093435849$

$Y_{n+3}-1298 Y_{n+2}+Y_{n+1}=0 ; \quad Y_{2}=3^{k} .233640, Y_{3}=3^{k} .303264540$

From (14) and (15), the values of $f_{n}$ and $g_{n}$ are found to be

$f_{n}=\frac{1}{3^{k}}\left(1298 X_{n+2}-4680 Y_{n+2}\right) ; g_{n}=\frac{1}{3^{k}}\left(1298 \sqrt{13} Y_{n+2}-360 \sqrt{13} X_{n+2}\right)$

\section{Properties}

1. When $k \equiv 0(\bmod 2), 6\left(1298 X_{2 n+3}-4680 Y_{2 n+3}+2.3^{2 k}\right)$ is a nasty number.

2. When $k \equiv 0(\bmod 3), 1298 X_{3 n+4}-4680 Y_{3 n+4}+3\left(1298 X_{n+2}-4680 Y_{n+2}\right)$ is a cubic integer.

\subsection{Choice 5: $t=2 k+5, k>0$}

The Pell equation is

$x^{2}=13 y^{2}-3^{2 k+5}$

Let $\left(X_{0}, Y_{0}\right)$ be the initial solution of (17) given by

$X_{0}=3^{k-1} .19 ; \quad Y_{0}=3^{k-1} .14$

Applying Brahmagupta lemma between $\left(X_{0}, Y_{0}\right)$ and $\left(\tilde{x}_{n}, \tilde{y}_{n}\right)$, the sequence of non-zero distinct integer solutions to (17) are obtained as

$$
\begin{gathered}
X_{n+1}=\frac{3^{k-1}}{2}\left(19 f_{n}+14 \sqrt{13} g_{n}\right) \\
Y_{n+1}=\frac{3^{k^{-1}}}{2 \sqrt{13}}\left(14 \sqrt{13} f_{n}+19 g_{n}\right)
\end{gathered}
$$

The recurrence relations satisfied by the solutions of (17) are given by

$X_{n+3}-1298 X_{n+2}+X_{n+1}=0 ; X_{1}=3^{k-1} .45091, X_{2}=3^{k-1} .58528099$

$Y_{n+3}-1298 Y_{n+2}+Y_{n+1}=0 ; Y_{1}=3^{k-1} .12506, Y_{2}=3^{k-1} .16232774$

From (18) and (19), the values of $f_{n}$ and $g_{n}$ are found to be

$f_{n}=\frac{1}{3^{k+6}}\left(325156 Y_{n+2}-90182 X_{n+2}\right) ; g_{n}=\frac{1}{3^{k+6}}\left(25012 \sqrt{13} X_{n+2}-90182 \sqrt{13} Y_{n+2}\right)$

The integer solutions presented in each of the sections 1 to 5 satisfy the following relations.

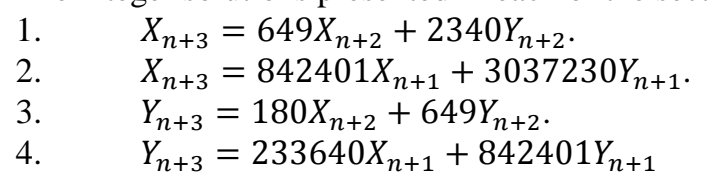

\section{Conclusion}

To conclude, one may search for other patterns of solutions to the similar equation considered above. 


\section{References}

[1] Ahmet Tekcan,Betul Gezer and Osman Bizin "On the integer solutions of the Pell equation $x^{2}-d y^{2}=2^{t}$ ”, World Academy of Science, Engineering and Technology, 1(2007) 522-526.

[2] Ahmet Tekcan "The Pell equation $x^{2}-\left(k^{2}-k\right) y^{2}=2^{t}$ ", World Academy of Science, Engineering and Technology, 19 (2008) 697-701.

[3] Gopalan.M.A. and R.S.Yamuna "Remarkable observations on the ternary quadratic equation $y^{2}=\left(k^{2}+1\right) x^{2}+1, k \in z-\{0\}$," Impact J.Sci. Tech., 4(4) (2010) 61-65.

[4] Gopalan.M.A. and R.Vijayalakshmi "Special Pythagorean triangles generated through the integral solutions of the equation $y^{2}=$ $\left(k^{2}+1\right) x^{2}+1 "$, Antarctica Journal of Mathematics, 7(5) (2010) 503-507.

[5] Gopalan.M.A. and A.Vijaya Sankar "Integral solutions of $y^{2}=\left(k^{2}-1\right) x^{2}-1$ ", Antarctica Journal of Mathematics, 8(6) (2011) 465-468.

[6] Gopalan.M.A. and B.Sivakami "Special Pythagorean triangles generated through the integral solutions of the equation $y^{2}=\left(k^{2}+2 k\right) x^{2}+$ 1", Diophantus Journal of Mathematics, 2(1) (2013) 25-30.

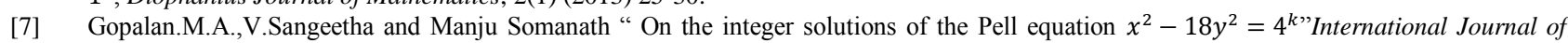
Engineering and Science Invention (IJESI), 2 (12) (2013) 01-03.

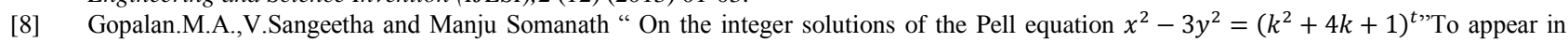
Proceedings of the International Conference on Mathematical Methods and Computation Jamal Mohamed College(Autonomous), Tiruchirappalli, India, February 2014.

[9] Jones.J.P. "Representation of solutions of Pell equations using Lucas sequences", Acta Academia Pead. Ag. Sectio Mathematicae, 30 (2003) 75-86.

[10] Kaplan.P. and K.SWilliams., "Pell's equation $x^{2}-m y^{2}=-1,-4$ and continued fractions", Journal of Number Theory, 23(1986) 169-182.

[11] Keskin.R. "Solutions of some quadratic Diophantine equations", Computers and Mathematics with Applications, 60(2010) 2225-2230.

[12] Lenstra.H.W. "Solving the Pell equation", Notices of the AMS, 49(2) (2002) 182-192.

[13] Matthews.K. "The Diophantine equation $x^{2}-D y^{2}=N, D>0$ ", Expositiones Math., 18(2000) 323-331.

[14] Tekcan.A. O.Bizin and M.Bayraktar "Solving the Pell equation using the fundamental elements of the Field $Q(\sqrt{\Delta})$," South East Asian Bulletin of Mathematics, 30 (2006) 355-366,

[15] Tekcan.A. "The Pell equation $x^{2}-D y^{2}= \pm 4 "$, Applied Mathematical Sciences, 1(8) (2007) 363-369. 